DOI: $10.12731 / 2306-1561-2013-4-12$

\title{
PROBLEM OF PRELIMINARY DESIGN PROCESSING EQUIPMENT
}

\author{
Ilyina M.V., Krasilnikov V.E., Nemtinov V.A.
}

Abstract

At present there are various approaches to the solution of a problem of processing equipment design. However, approaches to preparatory design not depending on a type of the equipment are not insufficiently studied. One of such approaches is representation of properties and device structure in the form of a tree structure, and design result in the form of a set of rules created on the basis of the trees presented.

Keywords: design, equipment, expert system, tree.

\section{УДК 004.891.2}

\section{ЗАДАЧА ПРЕДВАРИТЕЛЬНОГО ПРОЕКТИРОВАНИЯ ТЕХНОЛОГИЧЕСКОГО ОБОРУДОВАНИЯ}

\section{Ильина М.В., Красильников В.Е., Немтинов В.А.}

\section{Аннотация}

В настоящее время существуют различные подходы $\kappa$ решению задачи проектирования технологического оборудования. Однако, подходы к предварительному проектированию не зависящие от вида оборудования не достаточно изучены. Одним из таких подходов является представление свойств и структуры аппарата в виде древовидной структуры, а результата проектирования в виде множества правил, созданных на основе представленных деревьев.

Ключевые слова: проектирование, оборудование, экспертная система, дерево

Конструкцию любого аппарата можно представить, как совокупность различных элементов, в которых множество элементов образуют более крупные элементы [1]. Это даёт возможность представить конструкцию аппарата в виде дерева, пример которой представлен на рисунке 1 . 


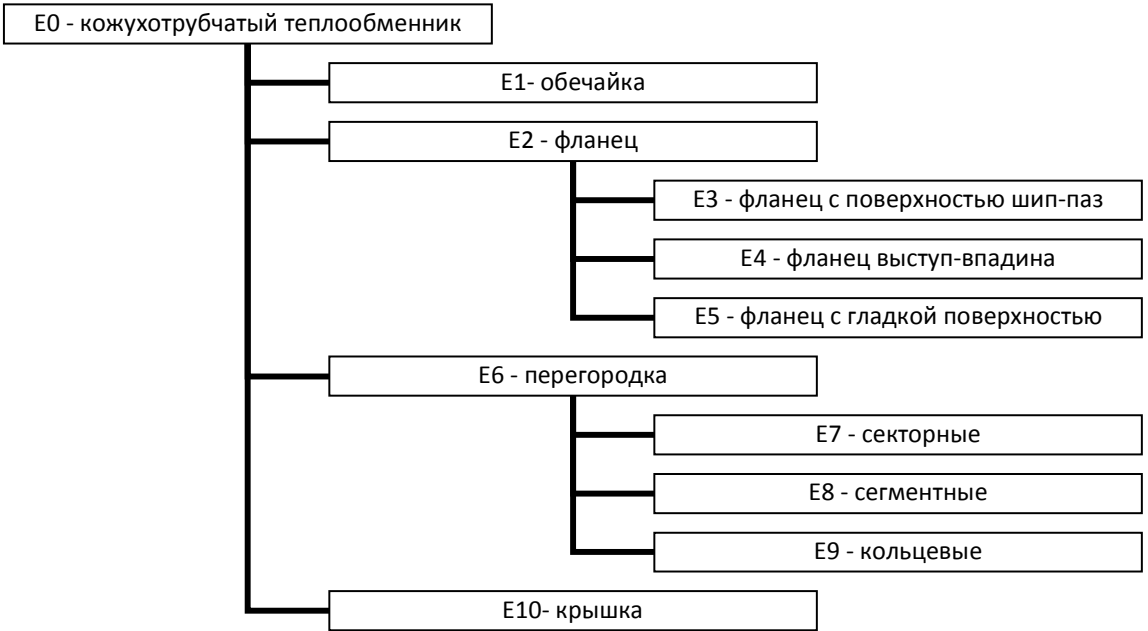

\section{Рисунок 1 - Пример части дерева вариантов конструкции кожухотрубчатого теплообменника}

EO - корень дерева. Корнем дерева может быть не только определенный вид аппарата, но и группа аппаратов, технологическая схема и производство. Более низкие уровни представляют собой детализацию вариантов набора элементов. Уровень детализации зависит от поставленной задачи и в пределе представляет собой конечные неделимые элементы оборудования.

Любой объект имеет характеристики, которые в свою очередь можно сгруппировать по различным критериям, это позволяет представить их в виде дерева (рисунок 2). Это могут быть характеристики различной меры, например геометрические, физические, временные и другие характеристики.

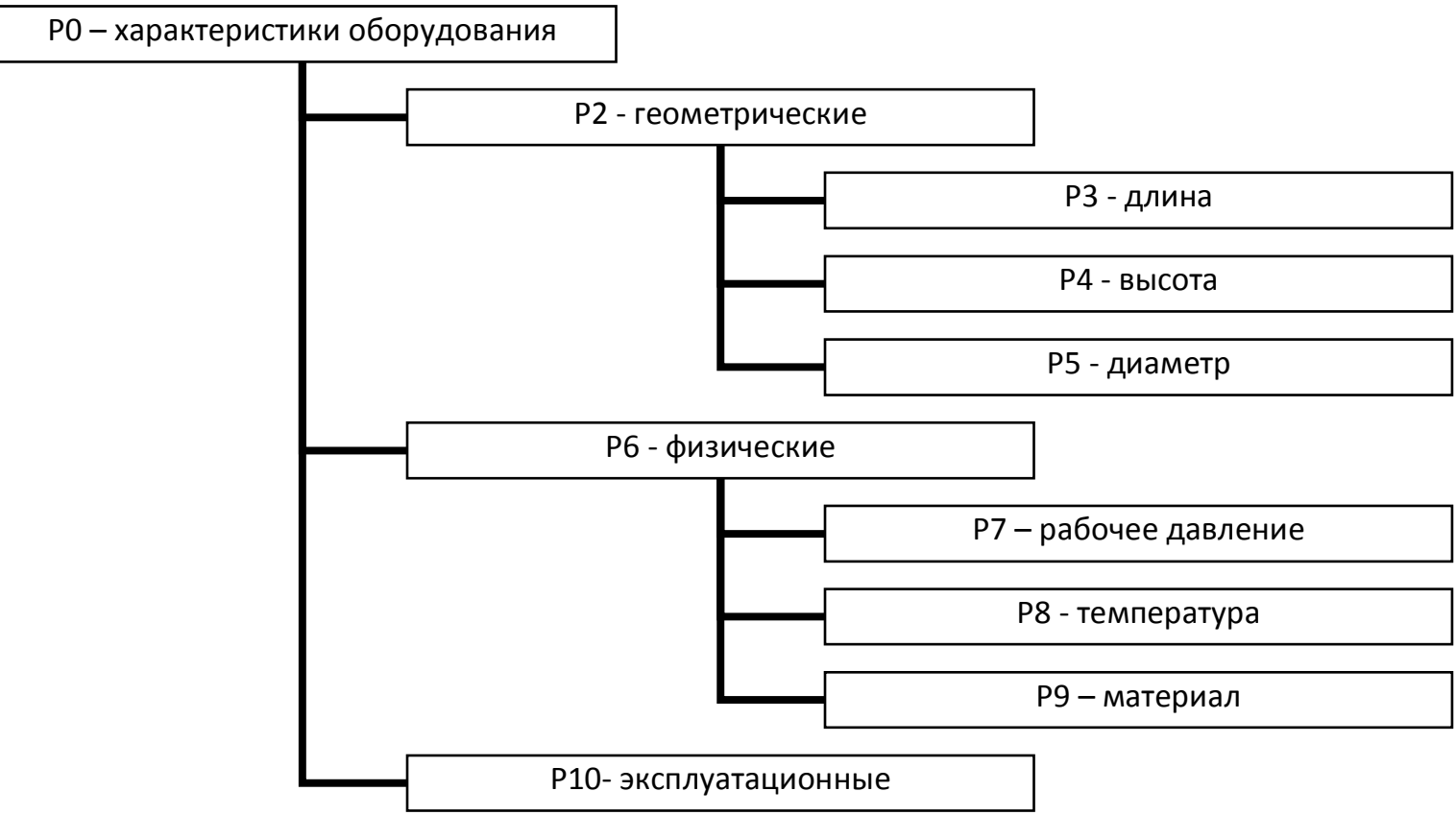

Рисунок 2 - Пример части дерева характеристик 
Решение задачи предварительного проектирования находится с помощью множества правил, сформированных из элементов деревьев описанных выше и значений этих элементов. Значения могут быть как целочисленные, так и представлять собой диапазоны или дискретные наборы. В простейшем случае элементы в правилах могут соединяться логической "и", но также могут быть более сложные варианты логических конструкций.

Точность решения зависит от числа входных данных. Чем больше исходных данных, тем большее количество правил будет применено и тем конкретнее будет решение. Чем меньше входных данных, тем меньше правил будет применено и результат решения будет более абстрактным.

Примеры правил:

$$
\begin{aligned}
& \text { Е3 и }(P 7=0.5 \text { или } P 11<10) \\
& \text { E10 и } P 6<10 \text { и P6 > } 2 \text { и P2 IN }(1,2,3,4)
\end{aligned}
$$

E3 и E7 и $P 3<8$

Таким образом, задачу предварительного проектирования любого технологического оборудования можно решать, представив элементы и характеристики проектируемого оборудования в виде деревьев, а возможные сочетания этих элементов и их значений в виде правил. Решение задачи находится путём решения полученных логических правил.

\section{Список информационных источников}

[1] Михалевич, В.С. Вычислительные методы исследования и проектирования сложных систем / В.С. Михалевич, В.Л. Волкович. - М.: Наука, 1982. - 286 с. 